\title{
Von der Kommission für Rechtsgeschichte Österreichs der ÖAW zur KRGÖ an der Universität Wien
}

Der letzte hier veröffentlichte Tätigkeitsbericht (BRGÖ 2012, 405-406) schloss mit dem - überaus positiven - Ergebnis einer Evaluation der Kommission für Rechtsgeschichte Österreichs (KRGÖ) im September 2011, der ihr eigentlich das Verbleiben an der ÖAW hätte sichern sollen. Nichtsdestoweniger beschloss diese in weiterer Folge, dass die KRGÖ mit 31. März 2012 ihres Personals und ihrer Sachmittel entkleidet und mit Ende des Jahres 2012 gänzlich aufgelöst werde. Damit ging eine fast 150 Jahre währende Tradition institutionalisierter rechtshistorischer Forschung an der ÖAW zu Ende. Auch wurde damit ein Netzwerk von österreichischen Rechtshistorikerinnen und Rechtshistorikern aus vier österreichischen Universitäten zerstört, und zwar ohne dass man damit Kosten eingespart hätte, zumal die Mitglieder stets ehrenamtlich tätig gewesen waren.

Für die bis dahin von der ÖAW, der Akademie der Wissenschaften zu Göttingen und dem FWF finanzierten Projekte und Mitarbeiter/innen der KRGÖ konnte indessen die Möglichkeit einer Fortsetzung gefunden werden: Nachdem das Wissenschaftsministerium eine hundertprozentige Deckung der bis dahin von der ÖAW getragenen jährlichen Kosten bis 31. Dezember 2015 garantiert hatte, erklärte sich die Universität Wien bereit, für den genannten Zeitraum sowohl die Projekte als auch, soweit dies möglich war, die Mitarbeiter zu übernehmen. Am Europatag, dem 9. Mai 2012, wurde die „KRGÖ an der Universität Wien“ als Abteilung des Instituts für Rechts- und Verfassungsgeschichte der Universität Wien gegründet; die Leitung übernahm der bisherige KRGÖ-Obmann ao.Univ.Prof. Dr. Thomas OlECHOWSKI. Nach Übertragung auch der Drittmittelprojekte an die Universität Wien setzte sich die KRGÖ aus insgesamt zehn, großteils teilzeitbeschäftigten Mitarbeiter/innen zusammen: Von der Universität Wien finanziert waren bzw. sind dies die Damen und Herren Univ.-Ass. Dr. Eva ORTLIEB (40 Stunden/Woche), Univ.-Ass. DDr. Christoph SCHMETTERer (8 Std/W), Univ.-Ass. Mag. Kamila Maria Staudigl-CieCHOWicz (zunächst 20 Std/W, ab 1. Mai 201335 Std/W), Projektmitarbeiterin Mag. Susanne GMOSER (freie Dienstnehmerin bis 30. April 2013, siehe noch unten) sowie die das Sekretariat betreuende Frau Katharina BERNOLD (20 Std/W). Vom FWF finanziert waren dies die Projektmitarbeiterinnen Dr. Tamara EHS (20 Std/W; ausgeschieden mit 15. November 2012), Mag. Ellen FrANKE (30 Std/W; ausgeschieden mit 30. September 2013) und Mag. Miriam GASSNER (20 Std/W; ausgeschieden mit 30. Oktober 2012), von der Göttinger Akademie finanziert waren bzw. sind dies die freien Dienstnehmerinnen Mag. YasminSybille RESCHER (ausgeschieden mit 30. April 2013) und Mag. Sandra WeIsS. Im Berichtszeitraum neu hinzu kam am 1. Jänner 2013 der FWF-Projektmitarbeiter Mag. Stefan WEDRAC (20 Std/W); seit 1. Mai 2013 war Mag. GMOSER 
als freie Dienstnehmerin auf Rechnung der Göttinger Akademie für die KRGÖ tätig und überdies ab 1. Juli 2013 auch als FWF-Mitarbeiterin an der KRGÖ angestellt (15 Std/W).

Die KRGÖ an der Universität Wien hat die Aufgabe übernommen, sämtliche Forschungsprojekte ihrer Vorgängerorganisation fortzusetzen; zusätzlich aber sind ORTLIEB, SCHMETTERER und STAUDIGL-CIECHOWICZ mit der Abhaltung von Lehrveranstaltungen für das Studium der Rechtswissenschaften an der Universität Wien betraut worden. Dass diese Lehrverpflichtung bei annähernd gleich bleibenden Ressourcen zu Lasten der Forschung gehen muss, liegt auf der Hand (vgl. aber Ex 5,9).

Die „neue“ KRGÖ präsentierte sich der Fakultät am 10. Oktober 2012 mit einer Veranstaltung am Wiener Juridicum, bei der sowohl der Fontes iuris-Band 22 als auch der BRGÖ-Band 2012/1 vorgestellt werden konnten; in einer von Univ.Prof. Dr. Miloš VEC (Wien) moderierten Podiumsdiskussion gingen Univ.-Prof. Dr. Matthias JeSTAEDT (Freiburg i.Br.), Univ.-Prof. Dr. Martin F. POlAscheK (Graz) und Univ.-Prof. Dr. Dr.h.c. Thomas WinkelbaueR (Wien) der im Zuge der "Transferierung“ aufgeworfenen Frage nach, wo - zwischen Rechts- und Geschichtswissenschaften - sich der "richtige“ Standort der Rechtsgeschichte befinde.

$\mathrm{Zu}$ den einzelnen Forschungsprojekten im Berichtszeitraum 2012/2013 ist Folgendes zu sagen:

Im Rahmen des Forschungsschwerpunktes "Reichshofrat" (RHR) konnte das von Hon.Prof. HR Dr. Leopold Auer geleitete FWF-Projekt P 20586 "Appellationen an den Reichshofrat (1519-1740)“ erfolgreich zu Ende geführt werden. Dieses Projekt strebte eine umfassende Untersuchung der Appellationen an den RHR an, der als zweites Höchstgericht - neben dem Reichskammergericht - an der Spitze des Instanzenzugs im Heiligen Römischen Reich deutscher Nation stand. Quellenbasis waren vor allem die im Haus-, Hof- und Staatsarchiv überlieferten Prozessakten aus dem für die Durch- setzung der Appellation entscheidenden Zeitraum zwischen 1519 und 1740, auf deren Grundlagen sowohl juristische als auch historische Fragen, nach Rechtsanwendung und Prozess ebenso wie nach der Rolle von Reichsoberhaupt und Reichsgericht in der Rechtswirklichkeit des territorial strukturierten Reichs, gestellt wurden. Im Rahmen des Projekts fand 2012 eine vielbeachtete Tagung statt, deren Ergebnisse in Band 2013/1 der BRGÖ publiziert wurden. Eine umfassende Monographie ist in Vorbereitung.

Fortgeführt wurden die Arbeiten an den Relationen des Reichshofrats Johann Adam zu Starhemberg sowie die Kooperationen mit der Akademie der Wissenschaften zu Göttingen (Langzeitprojekt zur Erschließung der Akten des Reichshofrates) sowie mit Universität Graz (FWF-Projekt zum Supplikenwesen unter Rudolf II.). Begonnen wurde mit einem neuen Projekt, welches die Stellung des RHR als oberstes Bücheraufsichtsorgan im Heiligen Römischen Reich in Zeitraum 1765-1806 untersuchen soll. Zentrale Fragestellung wird hier sein, ob und wie sich Josephinismus und Französische Revolution auf die Reichspressepolitik auswirkten und welche Rolle der RHR dabei spielte.

Im Rahmen des Forschungsprojekts „Kelsens Leben in Amerika (1940-1973) und die weltweite Verbreitung seiner Rechtslehre" (FWF-Projekt P 23747) unternahmen OLECHOWSKI, EHS, GASSNER und WEDRAC eine Reihe von Forschungsreisen, und zwar nach Washington D.C., New York, Harvard und Berkeley, ferner nach Rio de Janeiro, Montevideo, Buenos Aires, La Plata und Mexico City sowie auch - mit finanzieller Unterstützung des Hans Kelsen-Instituts - nach Moskau und Rijeka. Dabei konnten Zeitzeugen interviewt und Archivmaterial gesammelt, Gespräche mit Kelsenforschern geführt sowie mehrere Vorträge gehalten werden. Die Ergebnisse wurden in verschiedenen Aufsätzen publiziert und werden auch in die Biographie über Hans Kelsen einfließen, an der OLECHOWSKI arbeitet. 
Im Rahmen der Quelleneditionsreihe „Fontes iuris“ erschien im Frühjahr 2013 mit tatkräftiger Unterstützung von ORTLIEB der Band 23: Johannes RAMHARTER, Aus den Ratsprotokollen der Stadt Tulln. Die genannten Protokolle und einige ergänzenden Quellen vermitteln ein authentisches Bild vom Alltag in einer landesfürstlichen Stadt, v.a. im 16. und 17. Jh.: Kriminalfälle, Marktangelegenheiten, ein Stadtbrand und vieles mehr sind Gegenstände des Editionsbandes.

Es war dies der letzte Band, der von der ",alten“ Kommission für Rechtsgeschichte Österreichs der ÖAW herausgegeben wurde. Ende 2012 beschlossen die bisherigen Kommissionsmitglieder - Hon.-Prof. HR Dr. Leopold AUER, emer. o. Univ.-Prof. Dr. Ursula FloßmanN, o Univ.-Prof. DDr. Herbert KALB, emer. o. Univ.-Prof. Dr. Dr.h.c. Gernot KOCHER, emer. o. Univ.-Prof. Dr. Herbert MATIS, ao. Univ.-Prof. Dr. Christian Neschwara, emer. o. Univ.-Prof. Dr. Dr.h.c.mult. Werner OGRIS, ao. Univ.-Prof. Dr. Thomas OlechowsKI, ao. Univ.-Prof. Dr. Ilse ReIter-Zatloukal, emer. o. Univ.-Prof. Dr. Dr.h.c. Gerhard THÜR, emer. o. Univ.-Prof. Dr. Gunter WESENER und Univ.-Prof. Dr. Dr.h.c. Thomas WinKelbaUer - die Fontes iuris als Herausgeberkollektiv weiterzuführen. Der Verlag Böhlau, das Layout sowie die Nummerierung der einzelnen Bände wurden beibehalten. Der erste Band, der nach diesem Umbruch erscheinen sollte, war der zweite Teil des Preßburger Protocollum Testamentorum (Fontes Iuris 21/2). Die Publikation erfolgte 2014; auch hierzu mehr im nächsten Jahresbericht.

Die KRGÖ war 2012/2013 an der Organisation dreier Fachtagungen mitbeteiligt: Vom 30. Mai bis 2. Juni 2012 fand am Wiener Juridicum das 18. Forum Junger Rechtshistoriker/innen statt, und zwar unter dem Generalthema „making things legal / recht [durch] setzen“. Von Seiten der KRGÖ waren STAUDIGL-CIECHOWICZ und SCHMETTERER im Organisationsteam. Die Beiträge wurden in Heft 2/2013 der BRGÖ publiziert, siehe dort für weitere Details zur Tagung.
Vom 15.-16. Oktober 2012 fand im Jüdischen Museum Wien ein Symposium zu der postum veröffentlichten Schrift Kelsens „Secular Religion" statt; Veranstalter war das Hans KelsenInstitut in Verbindung mit der KRGÖ. Die dort gehaltenen Vorträge wurden fast genau ein Jahr später in der Schriftenreihe des Hans KelsenInstituts als Band 34 veröffentlicht.

Am 24. Oktober 2013 fand im Senatssaal der Universität Wien die Tagung „Von Wien nach Czernowitz. Rechts- und Staatswissenschaftliche Karrierewege um 1918“ statt. Diese gemeinsam mit dem Forum Zeitgeschichte der Universität Wien und dem Hans Kelsen-Institut organisierte Veranstaltung ging aus einem FWF-Projekt zur Geschichte der Wiener Rechts- und Staatswissenschaftlichen Fakultät 1918-1938 hervor, das 2009-2013 unter der Leitung von OLECHOWSKI und Mitarbeit von EHS und STAUDIGLCIECHOWICZ durchgeführt wurde. Die Tagungsbeiträge sollen in Heft 2/2014 der BRGÖ veröffentlicht werden; siehe dort für weitere Details.

Die budgetäre Situation der KRGÖ stellte sich in den Jahr 2012 und 2013 wie folgt dar:

Die ÖAW finanzierte die Monate Jänner bis März mit einem Personal- und Sachbudget in Höhe von $€ 33.900,00$; die Universität Wien stellte für die Monate April bis Dezember Mittel in Höhe von $€ 111.700,89$ bereit (von denen $€ 103.040,81$ verbraucht und der Rest in Rücklagen umgewandelt wurde). Dazu kamen $€ 93.348,40$ vom FWF und $€ 40.668,44$ von der Akademie der Wissenschaften zu Göttingen. Der Drittmittelanteil 2012 betrug somit 49,5\%.

Im Jahr 2013 erhielt die KRGÖ von der Universität Wien ein Personal- und Sachbudget in Höhe von $€ 154.519$,90, von denen $€ 150.923$,37 verbraucht wurden. Dazu kamen erneut Drittmittel im Ausmaß von $€ 86.086,05$ vom FWF und $€ 36.048,80$ von der Akademie der Wissenschaften zu Göttingen. Der Drittmittelanteil 2013 betrug somit $44,7 \%$.

Thomas OLECHOWSKI 